\title{
Abdominal distention and peritoneal effusion in a female Swiss albino mouse
}

\author{
Kemal Kosemehmetoglu, $M D^{1}$, Nadir Ulu, $M D^{2}$, Alper B. Iskit, $M D^{2}$, Pinar F. Arıkan, $M D^{1}$, Cenk Sokmensuer, $M D^{1}$, \\ Rustu Onur, MD, $P h D^{2}$ \& M. Oguz Guc, MD, $P h D^{2}$
}

During a routine examination of our department's animal husbandry unit, we found a female Swiss albino mouse with an extremely distended abdomen (Fig. 1). This 6-month-old mouse, which was socially housed with other female mice, weighed $83 \mathrm{~g}$. The average weight of a 6-month-old Swiss albino mouse in our colony is $35-40 \mathrm{~g}$. Upon examination, the animal was lethargic and its muzzle, oral mucous membranes, eyes, paws, and tail were pale. Other mice in the cage appeared normal. We had never encountered a similar presentation.

We euthanized the mouse by overdose of general anesthesia and carefully incised the abdominal skin and stretched it laterally to examine the abdominal wall (Fig. 2). Through the translucent stretched abdominal wall, we saw a large volume of reddishbrown peritoneal fluid. Before entering the abdominal cavity, we withdrew $35 \mathrm{ml}$ of fluid by syringe. Cytological evaluation of the peritoneal fluid showed that it predominantly consisted of lymphocytes, macrophages, and reactive mesothelial cells; few neutrophils were present in the fluid (Fig. 3).

We then examined the abdominal and thoracic cavities. There was no organomegaly nor any gross pathological changes except for a tan-to-brown discoloration of the liver. We removed the heart, lungs, spleen, liver, kidneys, stomach, and duodenum and placed them in 10\% neutralbuffered formalin. Tissue sections were prepared from samples of fixed tissue using routine procedures.

Histopathological examination revealed a diffuse acute inflammation of the viscera and

Departments of ${ }^{1}$ Pharmacology and ${ }^{2}$ Pathology, Hacettepe University Faculty of Medicine, 06100, Ankara, Turkey. Correspondence should be addressed to A.B.I. (alperi@hacettepe.edu.tr). peritoneal membranes that we diagnosed as acute peritonitis. Inflammatory infiltrates composed of neutrophils and lymphocytes were prominent in the parenchyma of the pancreas, kidneys, gallbladder, and hilus of the liver. Inflammatory cells were present on the serosal surface of all intra-abdominal organs examined (including stomach, intestines, and spleen), although the parenchyma of these organs was normal.

We also saw multifocal inflammatory infiltrates and necrotic pancreatic acini throughout the pancreatic lobules. There was no inflammation or enlargement of the common bile duct as it traversed through the pancreas. However, microscopic examination of the proximal (non-pancreatic)

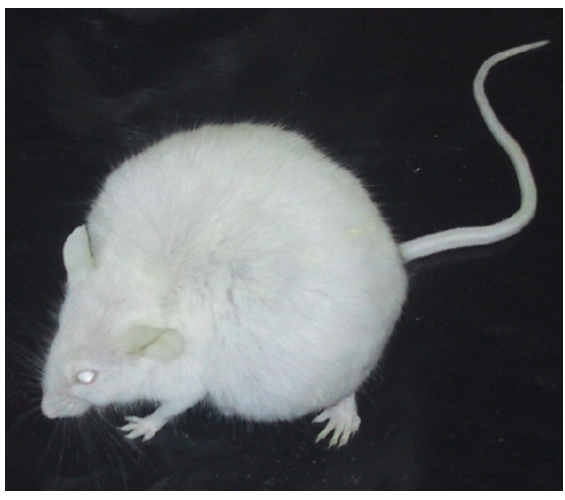

FIGURE 1 | A 6-month-old female Swiss albino mouse with an extremely distended abdomen. The mouse weighed $83 \mathrm{~g}$, more than twice the average weight of similarly aged animals in the colony.

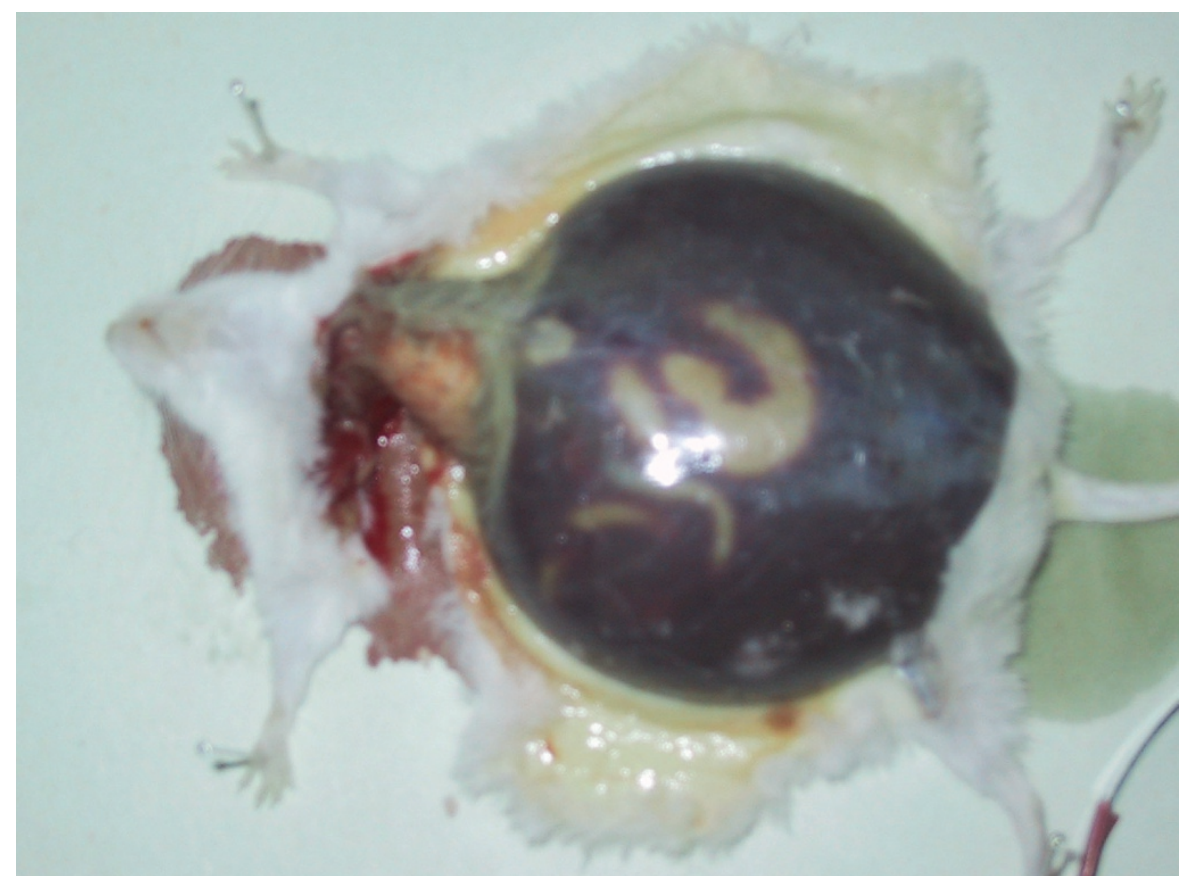

FIGURE 2 | The mouse shown in Figure 1 at necropsy. The skin has been reflected back revealing reddish-brown peritoneal fluid through the translucent stretched abdominal muscles. 


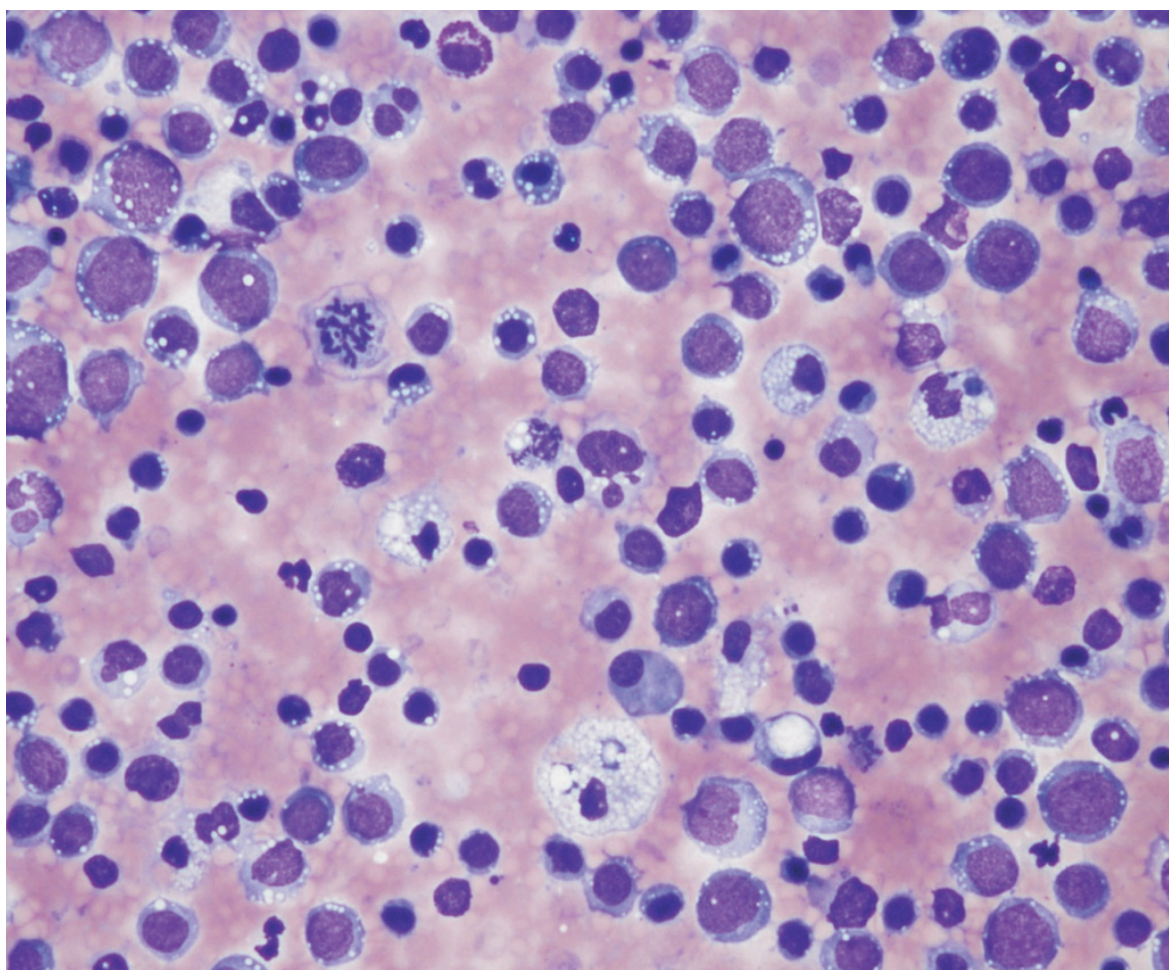

FIGURE 3 | Giemsa-stained sediment of peritoneal fluid seen in Figure 2. The cytology reveals a chronic, non-suppurative, mixed mononuclear reaction composed of lymphocytes, macrophages, and reactive mesothelial cells. The mitotic figure is a reactive mesothelial cell. $(\times 400)$.

biliary system revealed acute inflammatory cells infiltrating the wall of the common bile duct. We noticed a mineralized area of yellow-brown pigment, resembling bile, surrounded by acute inflammatory cells, in the hilus of the liver. At higher magnification, we saw that neutrophils had infiltrated the gallbladder wall and few mucosal epithelial cells remained.

Based on the clinical signs, cytology, and histopathologic description, what do you think caused the severe abdominal distention and peritoneal effusion seen in this mouse? How common is this condition?

\section{What's your diagnosis?}

